\section{Efectos del confinamiento por COVID-19 en la salud mental de estudiantes de educación superior en Chile}

\author{
RAFFAELA CARVACHO ${ }^{1, a}$, JAVIER MORÁN-KNEER ${ }^{1,2, b}$, \\ CLAUDIA MIRANDA-CASTILLO ${ }^{1,3, \mathrm{~b}}$, \\ VIRGINIA FERNÁNDEZ-FERNÁNDEZ ${ }^{4, b}$, \\ BEATRIZ MORA $^{c}$, YASNNI MOYA ${ }^{c}$, VÍCTOR PINILLA ${ }^{c}$, \\ IGNACIO TORO ${ }^{\circ}$, CONSTANZA VALDIVIA $^{\mathrm{c}}$
}

\section{Effects of the COVID-19 confinement on mental health among higher education students in Chile}

Background: Chilean higher education students have faced highly stressful events in 2020, including confinement and remote education. This population is particularly susceptible to mental health problems, such as high levels of stress, anxiety, and depression. Aim: To evaluate possible negative impacts of confinement by COVID-19 on Chilean higher education students' mental health. Material and Methods: The Center for Epidemiologic Studies Depression Scale (CES-D) Scale and the Profile of Mood States (POMS) were applied to 315 students during 2016 and to 301 students during 2020. Results: In 2020, depression mean scores for men (24.1) and women (29.7) exceeded the cutoff point, therefore suggesting the presence of depressive disorder. Women had higher depressive and anxious symptoms in both years, however, in 2020 a significant increase was observed for depression, indicating interaction between the year of assessment and gender $(\beta=6.74 ; p<.001)$. In both samples, first-year students had significantly higher depression scores $(\beta=-1,909 ; p=0.05)$. Conclusions: An increase in depression was observed in 2020. Confinement by COVID-19 had a high impact on mental health in female students. Women and first-year students appear to be high risk groups for developing depressive symptoms.

(Rev Med Chile 2021; 149: 339-347)

Key words: Anxiety; COVID-19; Depression; Students.

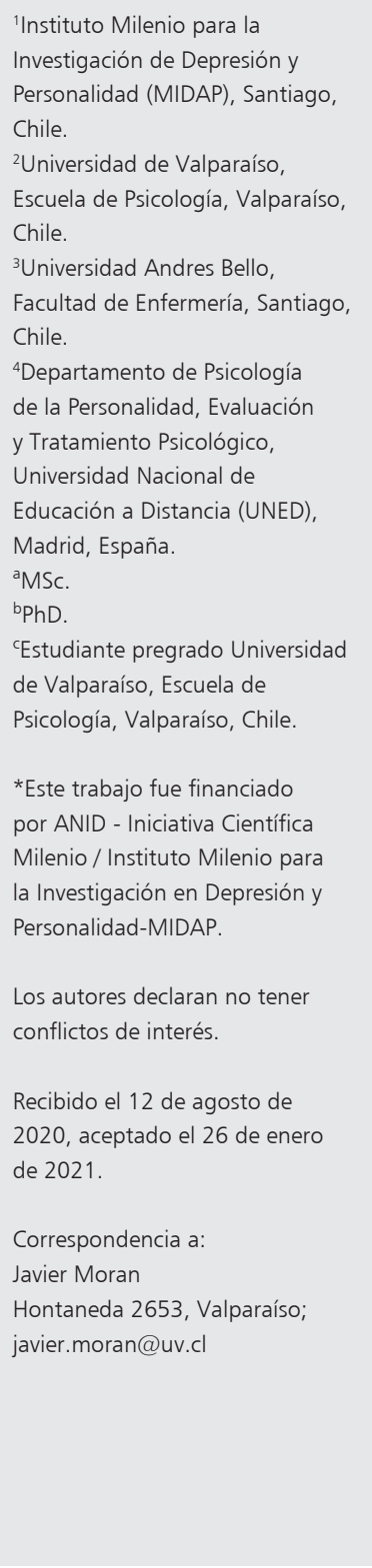

'Instituto Milenio para la

Chile.

${ }^{2}$ Universidad de Valparaíso,

Escuela de Psicología, Valparaíso,

Chile.

Facultad de Enfermería, Santiago, Chile.

${ }^{4}$ Departamento de Psicología de la Personalidad, Evaluación

y Tratamiento Psicológico

Universidad Nacional de

Educación a Distancia (UNED)

Madrid, España.

$\mathrm{MSC}$

'Estudiante pregrado Universidad de Valparaíso, Escuela de

Psicología, Valparaíso, Chile.

*Este trabajo fue financiado por ANID - Iniciativa Científica Milenio / Instituto Milenio para la Investigación en Depresión y

Los autores declaran no tener conflictos de interés.

Recibido el 12 de agosto de 2020, aceptado el 26 de enero

Javier Moran Hontaneda 2653, Valparaíso; javier.moran@uv.c
L a pandemia de COVID-19 ha significado una crisis para el sistema sanitario y económico, con sobrecarga de servicios y el establecimiento de acciones de confinamiento voluntario u obligatorio ${ }^{1,2}$. Según la literatura, el confinamiento puede generar consecuencias negativas en la salud mental de la población general, observándose un aumento de síntomas de estrés, estrés post-traumático (TEPT), ansiedad y depresión ${ }^{3,4}$. La evidencia reciente también parece indicar que ser más joven actúa como factor de riesgo para desarrollar problemas de salud mental asociados al COVID- $19^{5}$.

En este contexto, las generaciones que actualmente cursan educación superior en Chile han enfrentado un número importante de eventos adversos. Por un lado, el ingreso a la educación universitaria ha sido marcado por un proceso 
de selección 2020 irregular, cuya aplicación de pruebas a lo largo del país fue afectada por manifestaciones sociales ${ }^{6}$. Luego, producto de la pandemia, dichos estudiantes han debido adaptarse repentinamente a nuevas condiciones educativas, accediendo a sus clases y evaluaciones a través de canales digitales. Es decir, en un contexto de incertidumbre económica y miedo al contagio, esta población ha seguido enfrentando las demandas normativas del rendimiento académico, a través de canales no acostumbrados. En este nuevo escenario, la mayoría de las instituciones de educación superior han intentado mitigar en paralelo las dificultades de sus estudiantes, sin necesariamente manejar suficiente información respecto a las condiciones y estado de salud de éstos ${ }^{7}$.

Con anterioridad a esta crisis epidemiológica, ya existía evidencia indicando que los estudiantes universitarios son un grupo vulnerable al desarrollo de trastornos en salud mental. Un estudio sobre universitarios chilenos evidenció una prevalencia de sintomatología depresiva (durante la última semana) de $28 \%{ }^{8}$, la cual es superior al promedio en población general chilena $(15,8 \%)$ según la Encuesta Nacional de Salud ${ }^{9}$. Además, este grupo presenta un alto nivel de estrés, ansiedad, abuso de sustancias y trastornos alimenticios ${ }^{10-12}$. En este contexto, existen subgrupos especialmente vulnerables; las mujeres tienden a evidenciar mayores prevalencias de sintomatología depresiva y ansiosa en comparación a hombres ${ }^{13,14}$. En adición, aquellos estudiantes más vulnerables en términos socioeconómicos enfrentan dificultades de inclusión, adaptación y retención en el sistema de estudios superiores ${ }^{15}$. Estos subgrupos han crecido en las últimas décadas, alcanzando un punto de inflexión entre los años 2008 y 2009, donde las mujeres comenzaron a superar sistemáticamente el número de hombres matriculados ${ }^{16}$. Asimismo, el mayor acceso por parte de sectores sociales populares se asocia al aumento de la matrícula. En 1990 la matrícula total en educación superior era de 245.561 alumnos, y en 2019 asciende a 1.194.311; es decir, en casi 30 años la matrícula en Chile se cuadriplicó ${ }^{17,18}$.

Frente a las vulnerabilidades previamente observadas en esta población, cabe preguntarse por su estado de salud con relación a la condición de confinamiento. Un estudio realizado en China ${ }^{19}$, evaluó a estudiantes universitarios un mes después del primer brote de COVID-19. Entre sus resultados, se observó una prevalencia de sintomatología depresiva que superaba el puntaje de corte en el $9 \%$ de los casos (un aumento leve, comparado al $8,8 \%$ observado en un estudio previo a la pandemia con una muestra similar ${ }^{20}$ ). Aquellos estudiantes que percibieron miedo extremo durante el brote, que vivían en zonas afectadas, y quienes cursaban su último año presentaron mayor riesgo de desarrollar TEPT y/o depresión ${ }^{19}$. Otra investigación ${ }^{21}$, identificó una prevalencia de $24,4 \%$ de sujetos con sintomatología ansiosa, de la cual un $0,9 \%$ experimentaba ansiedad severa. Además, se determinaron factores protectores frente a la ansiedad, como la estabilidad de ingresos, vivir en áreas urbanas, y vivir con los padres; mientras que conocer gente contagiada de COVID-19 actuó como factor de riesgo. No se observó una diferencia significativa entre hombres y mujeres en relación a su nivel de ansiedad ${ }^{21}$. Ahora bien, estos resultados provienen de muestras con características culturales y sociales diferentes de la realidad chilena. En ese sentido, se requiere mayor evidencia frente a este fenómeno tan reciente, especialmente en jóvenes (de quienes aún se maneja poca información empírica $)^{22}$.

Considerando la gran magnitud de la población de estudiantes de educación superior en Chile, el impacto psicológico de la pandemia de COVID-19 sobre ellos se considera un asunto de salud pública. Esta población representa las fuerzas de trabajo y conocimiento técnico disponibles para resolver los desafíos colectivos de las próximas décadas. Asimismo, contar con datos sobre la salud mental de este grupo podría ayudar a Instituciones de educación superior, sanitarias y sociales a prevenir, evaluar e intervenir sobre las posibles consecuencias psicológicas negativas de la pandemia en sus estudiantes ${ }^{23}$.

Por consiguiente, este estudio se propone evaluar posibles impactos negativos del confinamiento por COVID-19 sobre la salud mental de estudiantes chilenos de educación superior. Específicamente, analizar efectos a nivel de sintomatología ansiosa y depresiva, así como identificar posibles grupos de riesgo en términos de género y tramo de la carrera. Frente a este objetivo, se hipotetiza que el estrés producto de esta pandemia y el confinamiento, podría aumentar la sintomatología de salud mental en los estudiantes de educación superior. 


\section{Método}

\section{Diseño}

Este estudio cuantitativo de corte transversal, busca establecer diferencias de medias en sintomatología ansiosa y depresiva en dos muestras independientes.

\section{Participantes}

La muestra se compuso por un total de 616 jóvenes, estudiantes de carreras técnicas y profesionales entre 18 y 25 años, pertenecientes a instituciones de educación superior de la región de Valparaíso. Fueron excluidos de la muestra estudiantes de postgrado.

Los datos fueron recogidos a partir de un muestreo no aleatorio en dos momentos: el primero corresponde a un estudio realizado el año 2016, que tuvo como objetivo comparar sintomatología ansiosa y depresiva en estudiantes chilenos de la región de Valparaíso respecto a un grupo de estudiantes españoles. A partir de los resultados de la muestra chilena, se generó una segunda medición durante junio del 2020, para evaluar las mismas variables en estudiantes que vivían en situación de confinamiento producto de la pandemia por COVID-19 en la región de Valparaíso. Para evaluar el confinamiento, se aplicó una encuesta de caracterización sociodemográfica que incluyó autorreporte del porcentaje de tiempo en confinamiento durante el último mes. Personas que reportaron menos de un $50 \%$ de tiempo en confinamiento, fueron excluidas.

\section{Procedimiento}

Para cada estudio se diseñó una encuesta, que incluyó antecedentes sociodemográficos y cuestionarios de screening clínico, que fueron difundidos en redes sociales a través de Google Forms durante noviembre de 2016 y junio de 2020, respectivamente. Previa a la aplicación del instrumento, se solicitó la firma electrónica de un consentimiento informado que asegura la confidencialidad de los datos.

Los procedimientos realizados respetaron normas éticas según la Declaración de Helsinki ${ }^{24}$. El estudio fue aprobado por dos entidades, los comités de ética de la Universidad Francisco de Vitoria de Madrid, y del Instituto Milenio para la Investigación de Depresión y Personalidad (MIDAP).

\section{Instrumentos}

Se aplicó una encuesta de datos sociodemográficos que consultó por género, edad, carrera, y año de ingreso a la carrera.

Para las variables clínicas se utilizaron los siguientes instrumentos:

1. Escala de Tensión del Perfil de Estados de Ánimo (POMS $)^{25}$. Una subescala del cuestionario POMS, de 9 ítems, evaluado en un continuo Likert de 5 puntos respecto a la frecuencia de aparición de síntomas ansiosos durante la última semana, incluido el día de hoy. Esta escala incluye adjetivos que reflejan incrementos en la tensión musculoesquelética: tenso, agitado, a punto de estallar, descontrolado, relajado, intranquilo, inquieto, nervioso y ansioso. La validación al español ${ }^{26}$ mostró un alpha de Cronbach de 0,79.

2. Escala de Depresión del Centro para Estudios Epidemiológicos (CES-D) ${ }^{27}$. Corresponde a un breve auto reporte de 20 ítems que evalúa la presencia de síntomas de depresión durante las últimas 2 semanas. Gempp y colegas ${ }^{28}$ presentaron una versión adaptada y estandarizada para población juvenil chilena, que mostró un índice de consistencia interna alfa $=0,87$.

\section{Análisis}

Se reportan frecuencias y descriptivos de tendencia central y dispersión para las variables sociodemográficas según año (2016 y 2020). Como forma de evaluar diferencias en la composión de ambos grupos se utilizó análisis de $\chi^{2}$ para variables de frecuencia y t de Student para comparar medias.

Se llevó a cabo un análisis de ANOVA factorial para evaluar la asociación entre variables género, tramo de carrera y las variables dependientes: sintomatología ansiosa y depresiva. Se presentan tamaños del efecto a partir del estadístico "d" de Cohen ${ }^{29}$ para pruebas de hipótesis en donde se evidencian valores $\mathrm{p}<0,05$.

Los análisis fueron realizados a través del software estadístico Jamovi ${ }^{\odot}$.

\section{Resultados}

Caracterización sociodemográfica de la muestra

La Tabla 1 resume las características sociodemográficas de la muestra en cuanto a edad, 
Tabla 1. Descripción de la muestra según años 2016 y 2020

\begin{tabular}{|c|c|c|c|}
\hline \multirow[t]{2}{*}{ Variables } & $\begin{array}{c}\text { Grupo "2016" } \\
(n=315)\end{array}$ & $\begin{array}{c}\text { Grupo "2020" } \\
(n=301)\end{array}$ & sig. \\
\hline & \multicolumn{2}{|c|}{ X (DE) / Frecuencia (\%) } & \\
\hline Edad & $21,1(2,03)$ & $21,0(1,75)$ & $t=0,784$ \\
\hline \multicolumn{4}{|l|}{ Género } \\
\hline Masculino & $125(40 \%)$ & $88(29 \%)$ & $\chi^{2}=7,43^{*}$ \\
\hline Femenino & $190(60 \%)$ & $213(71 \%)$ & \\
\hline \multicolumn{4}{|l|}{ Área de Carrera } \\
\hline Salud & $42(13,38 \%)$ & $59(19,67 \%)$ & $\chi^{2}=28,4^{* * *}$ \\
\hline Cs.Sociales & $120(38,22 \%)$ & $82(27,33 \%)$ & \\
\hline Humanidades & $18(5,73 \%)$ & $11 \quad(3,67 \%)$ & \\
\hline Administración y comercio & $19(6,05 \%)$ & $30(10 \%)$ & \\
\hline Ingenierías & $91(28,98 \%)$ & $82(27,33 \%)$ & \\
\hline Biología y Cs. Naturales & $10 \quad(3,18 \%)$ & $6(2 \%)$ & \\
\hline Artes, diseño y arquitectura & $7 \quad(2,23 \%)$ & $4 \quad(1,33 \%)$ & \\
\hline Técnicas & $0 \quad(0 \%)$ & $6(2 \%)$ & \\
\hline Pedagogías & $7 \quad(2,23 \%)$ & $20(6,67 \%)$ & \\
\hline Cantidad de años en la carrera & $2,02(1,64)$ & $2,24(1,50)$ & $t=-1,770$ \\
\hline \multicolumn{4}{|l|}{ Tramo de la carrera } \\
\hline $1^{\text {er }}$ año & $64(20,3 \%)$ & $36(12,1 \%)$ & $\chi^{2}=8,06^{*}$ \\
\hline Otros años & $187(59,5 \%)$ & $205(68,1 \%)$ & \\
\hline Último año & $64(20,3 \%)$ & $60(19,8 \%)$ & \\
\hline
\end{tabular}

Nota. * $p<0,05,{ }^{* *} p<0,01,{ }^{* * *} p<0,001, U=U$ de Mann Whitney test, $M=$ Media; $D E=$ Desviación Estándar.

género, área de la carrera, cantidad de años en la carrera y el tramo que cursan (primer año, otros años, último año). Al comparar ambos grupos, se observaron diferencias en la distribución de frecuencias, no así en las medias de edad y años de permanencia en la carrera. Es importante destacar que se excluyó de este análisis a personas que se identificaron con género no binario $(3,6 \%)$, debido a que esta opción solamente se consultó en la muestra 2020.

\section{Sintomatología ansiosa y depresiva}

La Tabla 2 muestra las medias y desviación estándar de sintomatología ansiosa y depresiva según género y tramo de carrera.

En cuanto a sintomatología ansiosa, solamente se observaron diferencias con relación al género como efecto principal ligado a la variable grupo. Tal como se aprecia en la Tabla 3 y Figura 1a, las mujeres obtuvieron medias de ansiedad más altas que los hombres $(\beta=1,289$; $\mathrm{p}=0,020)$ en las dos muestras. La comparación de efectos simples permite observar un tamaño del efecto "pequeño" $(\mathrm{d}=0,046)$ entre hombres y mujeres en la muestra 2016 y pequeño-mediano $(d=0,48)$ en 2020.

Respecto a sintomatología depresiva, se observaron diferencias a nivel de efecto principal tanto para género $(\beta=2,240 ; p=0,001)$, donde las mujeres mostraron medias más altas $(\mathrm{d}=0,31)$; como para grupo $(\beta=5,564 ; p<0,001)$, evidenciándose un aumento en la sintomatología depresiva en el grupo 2020, con un tamaño del efecto grande $(\mathrm{d}=0,84)$. Ambas variables mostraron un efecto de interacción $(\beta=6,74 ; \mathrm{p}<0,001)$ que es posible apreciar gráficamente en la Figura 1c. En términos de efecto simple, se observó un tamaño del efecto pequeño $(d=0,016)$ asociado al género en la muestra 2016 y un tamaño del efecto mediano $(d=0,63)$ en 2020. 
Tabla 2. Descripción de medias y desviación estándar para sintomatología ansiosa y depresiva en sexo y tramo de carrera para muestra 2016 y muestra 2020

\begin{tabular}{|c|c|c|c|c|c|c|}
\hline \multirow{2}{*}{$\begin{array}{l}\text { Variable } \\
\text { dependiente }\end{array}$} & \multirow[b]{2}{*}{ Grupo } & \multicolumn{2}{|c|}{ Género (M/DE) } & \multicolumn{3}{|c|}{ Tramo carrera (M/DE) } \\
\hline & & Hombre & Mujer & Primer año & Otros años & Último año \\
\hline \multirow{2}{*}{$\begin{array}{l}\text { Sintomatología } \\
\text { Ansiosa }\end{array}$} & 2016 & $14,5 / 7,55$ & 14,9 / 8,03 & $15,6 / 7,77$ & $14,4 / 7,75$ & $15,0 / 8,31$ \\
\hline & 2020 & $12,8 / 4,65$ & $15 / 4,56$ & $15,4 / 4,57$ & $14,1 / 4,52$ & $14,8 / 5,21$ \\
\hline \multirow{2}{*}{$\begin{array}{l}\text { Sintomatología } \\
\text { Depresiva }\end{array}$} & 2016 & $21,9 / 6,90$ & $20,7 / 7,22$ & $22,0 / 6,89$ & $21,1 / 6,92$ & $20,7 / 7,94$ \\
\hline & 2020 & $24,1 / 8,51$ & $29,7 / 9,07$ & $30,4 / 10,2$ & $27,6 / 9,02$ & $28,8 / 8,96$ \\
\hline
\end{tabular}

Nota. $M=$ Media; $\mathrm{DE}=$ Desviación estándar.

Tabla 3. ANOVA factorial para sintomatología ansiosa y depresiva en grupos 2016-2020 de acuerdo a sexo y tramo de carrera

\begin{tabular}{|c|c|c|c|c|}
\hline Variables & B & \multicolumn{2}{|c|}{$95 \% \mathrm{Cl}$} & $\mathbf{p}$ \\
\hline \multicolumn{5}{|l|}{ Sintomatología ansiosa } \\
\hline (Intercepto) & 14,311 & 13,768 & 14,855 & $<0,001$ \\
\hline Sexo (mujer) & 1,289 & 0,202 & 2,375 & 0,020 \\
\hline Grupo (2020) & $-0,772$ & $-1,858$ & 0,315 & 0,165 \\
\hline Sexo * Grupo & 1,853 & $-0,320$ & 4,026 & 0,095 \\
\hline \multicolumn{5}{|l|}{$R^{2}=0,013$} \\
\hline (Intercepto) & 148,835 & 14,26 & 15,512 & $<0,001$ \\
\hline Grupo (2020) & $-0,2435$ & $-1,5$ & 1,013 & 0,704 \\
\hline Tramo carrera 1 (otros años - primer año) & $-12,046$ & $-2,69$ & 0,281 & 0,112 \\
\hline Tramo carrera 2 (ultimo año - primer año) & $-0,5817$ & $-2,35$ & 1,188 & 0,520 \\
\hline Grupo 1 * Tramo carrera 1 & $-0,0853$ & $-3,06$ & 2,886 & 0,955 \\
\hline Grupo 1 * Tramo carrera 2 & 0,0619 & $-3,48$ & 3,6 & 0,973 \\
\hline \multicolumn{5}{|l|}{$\mathrm{R}^{2}=0,006$} \\
\hline \multicolumn{5}{|l|}{ Sintomatología depresiva } \\
\hline (Intercepto) & 24,11 & 23,431 & 24,79 & $<0,001$ \\
\hline Sexo (mujer) & 2,24 & 0,879 & 3,60 & 0,001 \\
\hline Grupo (2020) & 5,64 & 4,274 & 7,00 & $<0,001$ \\
\hline Sexo * Grupo & 6,74 & 4,014 & 9,46 & $<, 001$ \\
\hline \multicolumn{5}{|l|}{$R^{2}=0,192$} \\
\hline (Intercepto) & 25,092 & 24,3 & 25,888 & $<0,001$ \\
\hline Grupo (2020) & 7,67 & 6,08 & 9,262 & $<0,001$ \\
\hline Tramo carrera 1 (otros años - primer año) & $-1,909$ & $-3,79$ & $-0,024$ & 0,048 \\
\hline Tramo carrera 2 (ultimo año - primer año) & $-1,465$ & $-3,71$ & 0,776 & 0,201 \\
\hline Grupo 1 * Tramo carrera 1 & $-1,907$ & $-5,68$ & 1,861 & 0,322 \\
\hline Grupo 1 * Tramo carrera 2 & $-0,283$ & $-4,77$ & 4,2 & 0,902 \\
\hline$R^{2}=0,160$ & & & & \\
\hline
\end{tabular}

Nota. $B=$ Coeficiente de regresión estandarizado; $I C=$ Intervalo de confianza; $R^{2}=$ Varianza explicada por el modelo. 


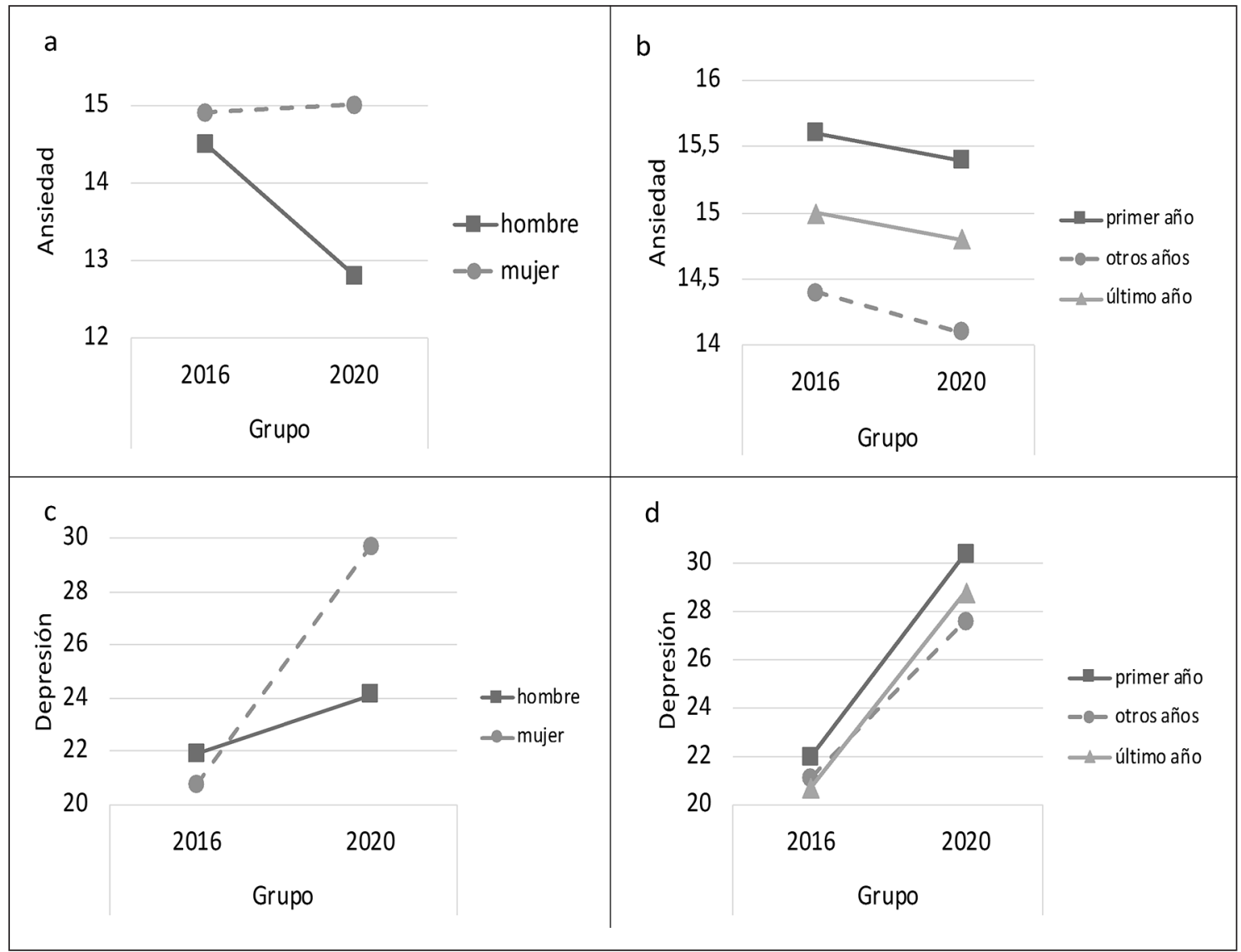

Figura 1. Medias de depresión y ansiedad según sexo y tramo de carrera para grupo 2016 y 2020.

En cuanto al análisis según tramo de carrera, solamente se observó un efecto principal $(\beta=-1,909 ; p=0,048)$, con puntajes más elevados de sintomatología depresiva para estudiantes de primer año respecto a otros años, y un tamaño del efecto pequeño, tanto en la muestra $2016(\mathrm{~d}=, 14)$ como en la muestra $2020(\mathrm{~d}=, 31)$.

\section{Discusión}

A partir de los resultados obtenidos, se evidencia un aumento preocupante de sintomatología depresiva en 2020. En 2016, ya se identifica una media bastante alta para hombres (21,9 pts.) y mujeres (20,7 pts.). Sin embargo, en 2020 el aumento es significativo, donde además tanto el promedio de hombres (24,1 pts.) como de mujeres (29,7 pts.) superan el puntaje de corte que sugiere la presencia de trastorno (24 pts.) en población juvenil ${ }^{28}$. Por el contrario, en el caso de la sintomatología ansiosa, no se observa una diferencia significativa entre las muestras del 2016 y 2020. Este desbalance del estado de ánimo depresivo por sobre el ansioso es discordante con la literatura, que señala que el estado de confinamiento, ser estudiante y ser joven, se relacionan tanto con un aumento de la ansiedad, como de depresión ${ }^{3,5,30}$. Sin embargo, en virtud de que la media propuesta para la escala de ansiedad del $\mathrm{POMS}^{26}$ es de 12,51 pts., es posible proponer que la sintomatología ansiosa, en realidad se ha mantenido elevada y por sobre la media.

En el caso de la sintomatología depresiva, es posible hipotetizar que su aumento podría estar asociado a emociones de desesperanza, debido a la interrupción de estudios, aislamiento social ${ }^{31}$ y los pronósticos de recesión económica ${ }^{32}$. Estos elementos podrían ser interpretados como un 
quiebre de proyecto vital para estos estudiantes universitarios, quienes se preparan para un futuro mercado laboral que se pronostica como difícil ${ }^{33}$ y que experimentan una vida universitaria que no responde a las expectativas comunes ${ }^{34}$. Asimismo, otra explicación posible es el efecto acumulativo de las manifestaciones sociales sobre la salud mental de los estudiantes, quienes han enfrentado experiencias complejas desde octubre del año 2019. Entre ellas, la interrupción del año académico, violencia al interior y fuera de establecimientos educacionales, y vulneración de Derechos $\mathrm{Hu}-$ manos $^{35}$.

La evidencia recomienda a universidades desarrollar intervenciones integrales que aborden el ambiente, currículum y directamente la salud mental de sus estudiantes, priorizando la prevención a través de medidas tempranas ${ }^{37,38}$. Los efectos aumentan cuando la población beneficiada presenta factores de riesgo significativos en pruebas de tamizaje ${ }^{39}$. Así, los resultados de este estudio reafirman la importancia de intervenir oportunamente con población femenina, quienes presentaron medias significativamente más altas de ansiedad en 2016 y 2020 en comparación a los varones. Y en cuanto a sintomatología depresiva, se evidencia una interacción entre el género y el efecto de la pandemia (modelo que explica casi un $20 \%$ de la varianza). Es decir, de la población de estudiantes universitarios que enfrentan el confinamiento, las mujeres resultan ser un grupo que se encuentra especialmente en riesgo.

Contrario a lo observado en estudiantes universitarios chinos ${ }^{19}$, fueron los estudiantes de primer año quienes mostraron mayor sintomatología depresiva. No obstante, dicho resultado es esperable, en cuanto esta transición vital de ingreso a la universidad demanda mayor adaptación, autonomía y exigencia académica ${ }^{36}$. Debido a que esta tendencia parece ser independiente del contexto de pandemia, estos resultados orientan hacia la necesidad de asignar recursos permanentes a este grupo de riesgo. Cabe destacar que las intervenciones basadas en internet presentan resultados significativos en reducir sintomatología depresiva, sin embargo, no es así en el caso de sintomatología ansiosa ${ }^{39}$. La efectividad de dichos resultados aumenta en intervenciones de duración moderada ( 4 a 8 semanas), con técnicas basadas en terapias cognitivo-conductuales (como mindfulness, identificar pensamientos disfuncionales, activación conductual, entre otros), y donde haya interacción humana (por ejemplo, los grupos de discusión generan mayor impacto que enviar infografías por correo electrónico $)^{39}$.

En cuanto a las limitaciones de la presente investigación, este es un estudio transversal, de muestreo por conveniencia, con reclutamiento en redes sociales. Por ello, los resultados no necesariamente reflejan la evolución de la sintomatología en el tiempo, ni tampoco la realidad de jóvenes sin acceso a internet. Además, los datos indagados en el 2020 fueron delimitados por los datos ya recogidos en 2016, de modo que no se pudo indagar en otras variables sociodemográficas.

Finalmente, los resultados obtenidos, si bien no son generalizables, entregan indicios que pueden orientar tanto a autoridades sanitarias como académicas, relevando el impacto a nivel de salud mental de la pandemia en un grupo que representa alrededor de 5\% de la población en nuestro país. El aumento pronunciado de la sintomatología depresiva y ansiosa urge a que se le dediquen recursos, a través del desarrollo de mayor investigación, acciones preventivas y de intervención a corto y mediano plazo, especialmente en mujeres y estudiantes de primer año, quienes fueron identificados como grupos de riesgo.

Agradecimientos: Este trabajo fue financiado por ANID - Iniciativa Científica Milenio / Instituto Milenio para la Investigación en Depresión y Personalidad-MIDAP.

\section{Referencias}

1. World Health Organization. WHO Director-General's opening remarks at the media briefing on COVID-19 [Internet]. World Health Organization. 2020; 4. Available from: https://www.who.int/dg/speeches/detail/ who-director-general-s-opening-remarks-at-the-mediabriefing-on-covid-19---22-june-2020.

2. The Lancet. COVID-19: remaking the social contract. Lancet [Internet]. 2020; 395 (10234): 1401. Available from: http://dx.doi.org/10.1016/S0140-6736(20)30983-1

3. Brooks SK, Webster RK, Smith LE, Woodland L, Wessely S, Greenberg N, et al. The psychological impact of quarantine and how to reduce it: rapid review of the evidence. Lancet [Internet]. 2020; 395 (10227): 91220. Available from: http://dx.doi.org/10.1016/S01406736(20)30460-8. 
4. Casagrande M, Favieri F, Tambelli R, Forte G. The enemy who sealed the world: Effects quarantine due to the COVID-19 on sleep quality, anxiety, and psychological distress in the Italian population. Sleep Med [Internet]. 2020; Available from: https://doi.org/10.1016/j. sleep.2020.05.011.

5. Hossain MM, Tasnim S, Sultana A, Faizah F, Mazumder $\mathrm{H}$, Zou L, et al. Epidemiology of mental health problems in COVID-19: a review. F1000Research. 2020; 9: 636.

6. Consejo de Rectores de las Universidades Chilenas. Acta sesión extraorinaria [Internet]. 2020; 1-9. Available from: file://C:/Users/Rafael/Documents/Revisiones y capítulos/covid universitarios/para intro/Acta_Sesion_Ext_1_2020.pdf.

7. Instituto Internacional para la Educación Superior en América Latina y el Caribe. COVID-19 y educación superior: De los efectos inmediatos al día después [Internet]. UNESCO. 2020; 44. Available from: http:// www.iesalc.unesco.org/wp-content/uploads/2020/04/ COVID-19-060420-ES-2.pdf.

8. Rossi JL, Jiménez JP, Barros P, Assar R, Jaramillo K, Herrera L, et al. Sintomatología depresiva y bienestar psicológico en estudiantes universitarios chilenos. Rev Med Chile 2019; 147 (5): 579-88.

9. Margozzini P, Passi A. Encuesta Nacional de Salud, ENS 2016-2017: Un aporte a la planificación sanitaria y políticas públicas en Chile Margozzini y Passi. ARS MEDICA Rev Ciencias Médicas 2018; (1): 30-4.

10. Morales G, Del Valle C, Belmar C, Orellana Y, Soto A, Ivanovic D. Prevalencia de consumo de drogas en estudiantes universitarios que cursan primer y cuarto año. Rev Med Chile 2011; 139 (12): 1573-80.

11. Jerez-Mendoza M, Oyarzo-Barría C. Estrés académico en estudiantes del Departamento de Salud de la Universidad de Los Lagos Osorno. Rev Chil Neuropsiquiatr. 2015; 53 (3): 149-57.

12. Baader TM, Rojas CC, Molina JLF, Gotelli M V., Alamo $\mathrm{CP}$, Fierro CF, et al. Diagnóstico de la prevalencia de trastornos de la salud mental en estudiantes universitarios y los factores de riesgo emocionales asociados. Rev Chil Neuropsiquiatr. 2014; 52 (3): 167-76.

13. Barrea-Herrera A, Neira-Cofré M, Raipán-Gómez $P$, Riquelme-Lobos P, Escobar B. Apoyo social percibido y factores sociodemográficos en relación con los síntomas de ansiedad, depresión y estrés en universitarios chilenos. Rev Psicopatol y Psicol Clin. 2019; 24 (2): 105-15.

14. Antúnez Z, Vinet E. Problemas de salud mental en estudiantes de una universidad regional chilena. Rev Medica chile. 2013; (141): 209-16.

15. Gallardo G, Lorca A, Morrás D, Vergara M. Experiencia de transición de la secundaria a la universidad de estu- diantes admitidos en una universidad tradicional chilena (CRUCH) vía admisión especial de carácter inclusivo. Pensam Educ Rev Investig Educ Latinoam. 2014; 51 (2): 135-51.

16. Consejo Nacional de Educación. Estadísticas de Género Matrícula Total 2015 [Internet]. 2015. Available from: https://www.cned.cl/sites/default/files/estadisticas_genero_matricula_total_2015.pdf

17. Rolando R, Salamanca J, Aliaga M. Evolución Matrícula Educación Superior de Chile Periodo 1990 - 2009 [Internet]. 2010. Available from: http://portales.mineduc. cl/usuarios/1234/File/Publicaciones/Estudios/5Estudio-Evolucion-Matricula-Historica-1990-2009.pdf

18. Servicio de Información de Educación Superior. Informe matrícula 2019 en Educación Superior en Chile [Internet]. 2019. Available from: https://www.cned.cl/ informes-en-es

19. Tang W, Hu T, Hu B, Jin C, Wang G, Xie C, et al. Prevalence and correlates of PTSD and depressive symptoms one month after the outbreak of the COVID-19 epidemic in a sample of home-quarantined Chinese university students. J Affect Disord [Internet]. 2020; 274 (March): 1-7. Available from: https://doi.org/10.1016/j. jad.2020.05.009.

20. Zhang Y, Liang W, Undergraduate ZC, Ms HZ. Validity and reliability of Patient Health Questionnaire-9 and Patient Health Questionnaire-2 to screen for depression among college students in China 2013; 5: 268-75.

21. Cao W, Fang Z, Hou G, Han M, Xu X, Dong J, et al. The psychological impact of the COVID-19 epidemic on college students in China. Psychiatry Res [Internet]. 2020; 287 (March): 112934. Available from: https://doi. org/10.1016/j.psychres.2020.112934.

22. Lee J. Mental health effects of school closures during COVID-19. Lancet Child Adolesc Heal [Internet]. 2020; 4 (6): 421. Available from: http://dx.doi.org/10.1016/ S2352-4642(20)30109-7.

23. Gruber J. Professors must support the mental health of trainees during the COVID-19 crisis. Science (80-) 2020 May 20.

24. Asociación Médica Mundial. Declaración de Helsinki de la AMM - Principios éticos para las investigaciones médicas en seres humanos [Internet]. World Medical Association, Inc. 2013. Available from: http://www.wma. net/es/30publications/10policies/b3/.

25. McNair D, Lorr M, Droppleman L. Manual for the profile of mood states (POMS). San Diego: Educational and Industrial Testing Service; 1971.

26. Andrade Fernández E, Arce Fernández C, Seaone Pesqueira G. Adaptación al español del cuestionario Perfil de los Estados de Ánimo en una muestra de deportistas. 
Psicothema [Internet]. 2002; 14 (4): 708-13. Available from: https://www.redalyc.org/articulo.oa?id=72714404

27. Radloff LS. The CES-D Scale: A Self-Report Depression Scale for Research in the General Population. Appl Psychol Meas. 1977; 1 (3): 385-401.

28. Gempp R, Avendaño C, Muñoz C. Normas y punto de corte para la Escala de Depresión del Centro para Estudios Epidemiológicos ( CES-D ) en población juvenil chilena. Ter Psicológica. 2004.

29. Cohen J. A power primer. Psychol Bull. 1992; 112 (1): 155-9.

30. Newby J, O’Moore K, Tang S, Christensen H, Faasse K. Acute mental health responses during the COVID-19 pandemic in Australia. medRxiv [Internet]. 2020; 2020.05.03.20089961. Available from: http://medrxiv. org/lookup/doi/10.1101/2020.05.03.20089961.

31. Meo SA, Abukhalaf AA, Alomar AA, Sattar K, Klonoff DC. COVID-19 pandemic: Impact of quarantine on medical students' mental wellbeing and learning behaviors. Pakistan J Med Sci. 2020 ;36 (COVID19-S4): S43-8.

32. Banco Central de Chile. Informe de política monetaria [Internet]. 2020. Available from: https://www.docdroid. net/bMkUQyx/ipom-marzo2020-pdf.

33. CEPAL. La Agenda 2030 para el Desarrollo Sostenible en el nuevo contexto mundial y regional: escenarios y proyecciones en la presente crisis [Internet]. Santiago; 2020. Available from: https://repositorio.cepal.org/ handle/11362/45336.

34. Fatima A, Utsav R. Stress in Students after Lockdown Due to COVID-19 Thereat and the Effects of Attending Online Classes. SSRN Electron J [Internet]. 2020; Preprint r. Available from: https://www.docdroid.net/ bMkUQyx/ipom-marzo2020-pdf.

35. Oficina del Alto Comisionado de Derechos Humanos. Informe sobre la misión a Chile [Internet]. 2019. Available from: https:/www.ohchr.org/Documents/Countries/ CL/Report_Chile_2019_SP.pdf.

36. Pérez M, Valenzuela M, Díaz A, González-Pienda J, Núñez J. Dificultades de aprendizaje en estudiantes universitarios de primer año. Atenea. 2013; 508: 135-50.

37. Fernández A, Howse E, Rubio-Valera M, Thorncraft $\mathrm{K}$, Noone J, Luu X, et al. Setting-based interventions to promote mental health at the university: a systematic review. Int J Public Health. 2016; 61 (7): 797-807.

38. Duffy A, Keown-Stoneman C, Goodday S, Horrocks J, Lowe M, King N, et al. Predictors of mental health and academic outcomes in first-year university students: Identifying prevention and early-intervention targets. BJPsych Open. 2020; 6 (3): 1-8.

39. Harrer M, Adam SH, Baumeister H, Cuijpers P, Karyotaki E, Auerbach RP, et al. Internet interventions for mental health in university students: A systematic review and meta-analysis. Int J Methods Psychiatr Res. 2019; 28 (2): 1-18. 